\title{
Design and on-orbit operation of the adiabatic demagnetization refrigerator on the Hitomi Soft X-ray Spectrometer instrument
}

\author{
Peter J. Shirron*a, Mark O. Kimball ${ }^{\mathrm{a}}$, Bryan L. James ${ }^{\mathrm{a}}$, Theodore Muench ${ }^{\mathrm{a}}$, Edgar R. \\ Canavan ${ }^{\mathrm{a}}$, Michael J. DiPirro, Thomas A. Bialas ${ }^{\mathrm{a}}$, Gary A. Sneiderman ${ }^{\mathrm{a}}$, Kevin R. \\ Boyce $^{a}$, Caroline A. Kilbourne ${ }^{a}$, Frederick S. Porter ${ }^{a}$, Richard L. Kelley ${ }^{a}$, Ryuichi \\ Fujimoto $^{\mathrm{b}}$, Yoh Takei ${ }^{\mathrm{c}}$, Seiji Yoshida ${ }^{\mathrm{d}}$, Kazuhisa Mitsuda ${ }^{\mathrm{c}}$ \\ ${ }^{a}$ NASA/Goddard Space Flight Center, Greenbelt, MD, USA 20771; ${ }^{\text {KKanazawa }}$ \\ University, Kanazawa, Ishikawa 920-1192, Japan; ' ISAS/JAXA, 3-1-1 Yoshino-dai, \\ Chuo-ku, Sagamihara, Kanagawa 252-5210, Japan; ${ }^{\mathrm{d}}$ Sumitomo Heavy Industries, \\ Ltd., Niihama, Ehime 792-8555, Japan
}

\begin{abstract}
The Soft X-ray Spectrometer instrument on the Astro-H observatory contains a 6x6 array of x-ray microcalorimeters that is cooled to $50 \mathrm{mK}$ by an adiabatic demagnetization refrigerator (ADR). The ADR consists of three stages in order to provide stable detector cooling using either a $1.2 \mathrm{~K}$ superfluid helium bath or a $4.5 \mathrm{~K}$ Joule-Thomson (JT) cryocooler as its heat sink. When liquid helium is present, two of the ADR's stages are used to single-shot cool the detectors while rejecting heat to the helium. After the helium is depleted, all three stages are used to continuously cool the helium tank (to about $1.5 \mathrm{~K}$ ) and single-shot cool the detectors (to $50 \mathrm{mK}$ ), using the JT cryocooler as its heat sink. The Astro-H observatory, renamed Hitomi after its successful launch in February 2016, carried approximately 36 liters of helium into orbit. On day 5 , the helium had cooled sufficiently $(<1.4 \mathrm{~K})$ to allow operation of the ADR. This paper describes the design, operation and on-orbit performance of the ADR, and the use of the ADR's heat rejection as a tool for mass gauging the helium tank.
\end{abstract}

Keywords: $x$-ray, astronomy, adiabatic demagnetization refrigerator, microcalorimeter

\section{INTRODUCTION}

The Soft X-ray Spectrometer (SXS) ${ }^{1}$ on the Hitomi observatory ${ }^{2}$ is a high-resolution imaging spectrometer designed to measure the energy of individual $\mathrm{x}$-ray photons in the 0.2 to $13 \mathrm{keV}$ band. Imaging is accomplished using a small (6x6) array of microcalorimeters ${ }^{3}$ located at the focal point of a grazing-incidence $x$-ray telescope ${ }^{4}$. Each pixel in the array can resolve photon energies to $4-5 \mathrm{eV}$. This resolution is achieved in part by cooling the array to 50 millikelvin. The cryogenic system ${ }^{5}$ consists of a 3 -stage adiabatic demagnetization refrigerator (ADR) connected to a 40-liter superfluid helium tank (at $<1.3 \mathrm{~K}$ ) and to a $4.5 \mathrm{~K}$ Joule-Thomson (JT) cryocooler with additional 2-stage Stirling cryocoolers ${ }^{6,7}$ used to cool vapor-cooled-shields and provide pre-cooling for the JT stage.

The ADR is configured so that it can use either the superfluid helium or the JT cryocooler as its heat sink. The operating strategy was to launch with 30+ liters of liquid helium and initially operate the ADR in "cryogen mode" where it rejects its heat directly to the helium. Operation would continue until the liquid helium was exhausted, then transition to a "cryogen-free mode" where the ADR assumes the additional task of cooling the (empty) helium tank while rejecting heat to the JT cryocooler. The ADR's design and performance in these two operating modes have been reported previously ${ }^{8,9,10}$, but changes to the cryogen-free mode algorithm made shortly before launch to improve its robustness are reported in a companion paper ${ }^{11}$. This paper presents on-orbit performance results for operation of the ADR with liquid helium present, as this was the only mode used during the 38 days in orbit before an anomaly ended the mission ${ }^{12}$.

*Peter.Shirron@nasa.gov; phone 1301 286-7327; fax 1301 286-0389 


\section{ADR DESIGN AND OPERATION}

\subsection{ADR Requirements and Design Summary}

Figure 1 shows a subset of the SXS cryogenic system, emphasizing the thermal connections between the ADR, the superfluid helium tank and the JT cryocooler. Figure 2 shows a solid model representation of the ADR assembly. The four heat switches (HS 1 to HS 4) are active gas-gap. The assembly is inserted into a well in the top end of the helium tank, with the central mounting plate serving as both a mechanical and thermal interface to the helium tank. Table 1 summarizes the design and operating parameters of the ADR.

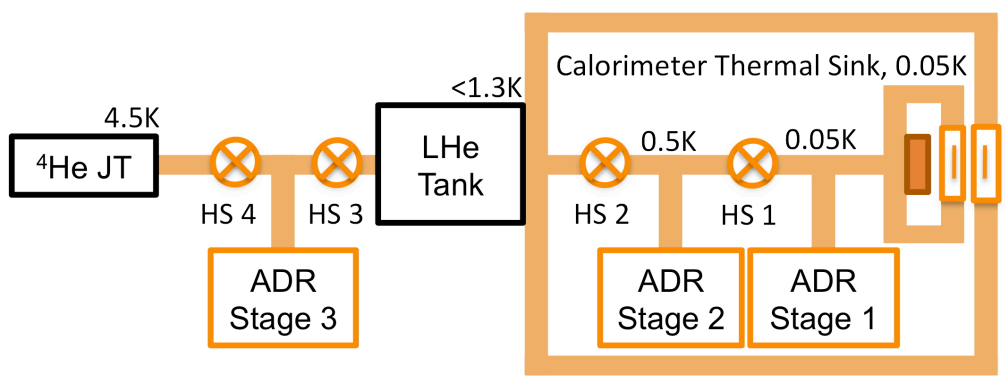

Figure 1. Thermal schematic of the subset of the Hitomi cryogenic consisting of the ADR, helium tank and JT cryocooler.

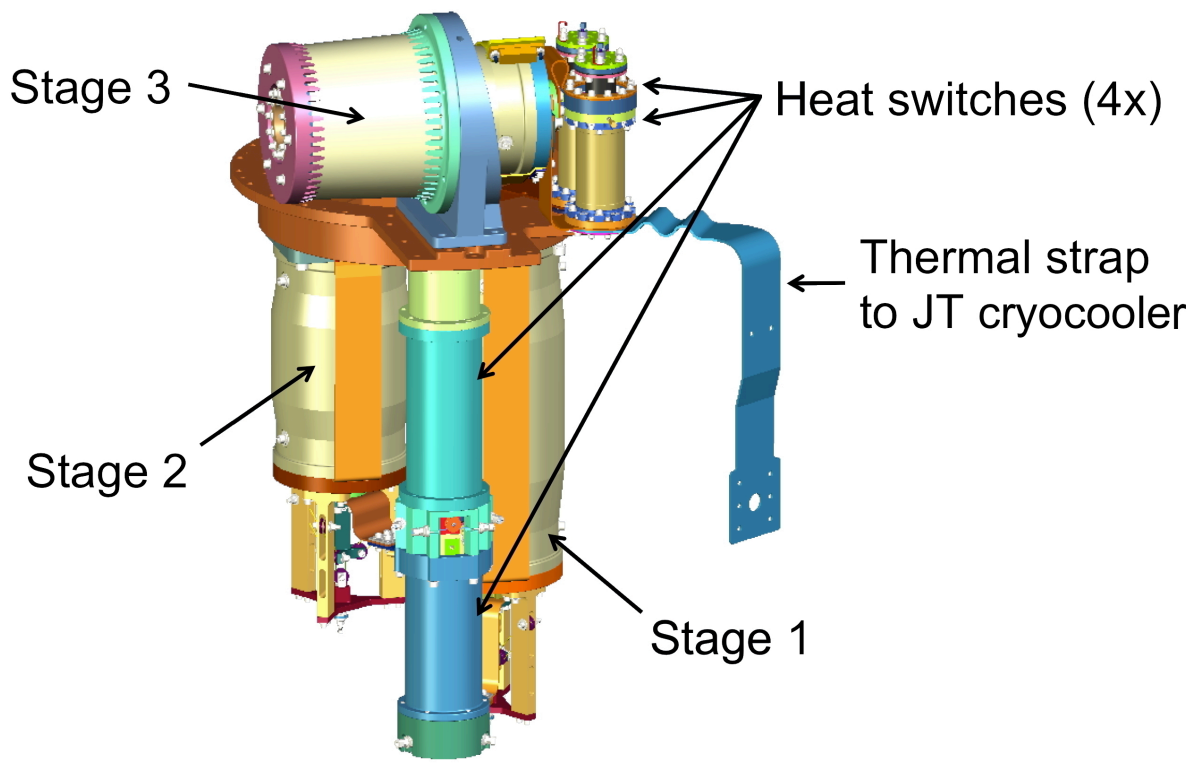

Figure 2. Solid model representation of the SXS 3-stage ADR.

When the ADR is operated using the helium as a heat sink, only stages 1 and 2 are needed to cool the detector array to $50 \mathrm{mK}$. The recycling process consists of the same sequence of steps regardless of starting condition (temperatures and magnet currents). The sequence and associated parameters, such as the temperature gradients applied across heat switches to drive heat flow, were optimized to yield the shortest recycle time at the expense of a small increase in the heat rejected to the helium tank. Even so, the time-average heat load on the helium of approximately $0.07 \mathrm{~mW}$ is still well below the allocation of $0.20 \mathrm{~mW}$ (needed to meet the 3 -year lifetime requirement). 
Table 1. Requirements, and design and operating parameters for the 3-stage SXS ADR.

\begin{tabular}{|c|c|c|c|}
\hline \multicolumn{4}{|c|}{ Performance Requirements } \\
\hline & Cryogen Mode & & gen-free Mode \\
\hline Detector operating temperature $(\mathrm{K})$ & 0.05 & & 0.05 \\
\hline Detector temperature stability ( $\mu \mathrm{K}$ rms) & 2.5 & & 2.5 \\
\hline Detector housing temperature stability $(\mathrm{mK})$ & 1.0 & & 1.0 \\
\hline Detector heat load $(\mu \mathrm{W})$ & 0.27 & & 0.47 \\
\hline Minimum observing efficiency & $90 \%$ & & $90 \%$ \\
\hline Typical hold time (hours) & 24 & & 15 \\
\hline Typical recycle time (hours) & 1 & & 2 \\
\hline Maximum heat sink load (mW) & 0.2 (avg) & & 30 (peak) \\
\hline Maximum magnetic field at detectors (mT) & 10 & & 10 \\
\hline \multicolumn{4}{|c|}{ Physical Design Parameters } \\
\hline & $\underline{\text { Stage } 1}$ & Stage 2 & Stage 3 \\
\hline Refrigerant type & $\mathrm{CPA}$ & GLF & GLF \\
\hline Refrigerant mass (g) & 270 & 147 & 147 \\
\hline Maximum magnetic field $(\mathrm{T})$ & 2 & 3 & 3 \\
\hline Maximum magnet voltage (V) & \pm 1 & \pm 1 & \pm 1 \\
\hline Magnet inductance $(\mathrm{H})$ & 250 & 200 & 200 \\
\hline \multicolumn{4}{|c|}{ Operating Parameters (Cryogen Mode) } \\
\hline & Stage 1 & Stage 2 & \\
\hline Hold temperature $(\mathrm{K})$ & 0.05 & 0.50 & \\
\hline Demagnetization temperature $(\mathrm{K})$ & 0.75 & 1.40 & \\
\hline Maximum reject temperature $(\mathrm{K})$ & 1.60 & 1.55 & \\
\hline
\end{tabular}

The ADR control electronics (ADRC) use independent 4-wire, 2-quadrant power supplies for regulating the current in each stage's magnet, and can exert either open-loop or closed-loop control. In open-loop control, fixed voltages are applied to the magnet, and in closed-loop control, magnet voltage is the output of a proportional-integral-derivative (PID) feedback loop. The ADR stages are nearly always in closed-loop control, with the constraints that magnet voltage is limited to $\pm 1 \mathrm{~V}$, and the current is between 0 and $+2.00 \mathrm{~A}$. Open-loop control is used only for stage 1 as it demagnetizes to its hold temperature, enabling the PID circuit to switch control thermometers from a Cernox ${ }^{\mathrm{TM}} 1030^{13}$ having good resolution at temperatures above about $0.3 \mathrm{~K}$ to a germanium resistance thermometer (GR-200A-30 ${ }^{13}$ ) preselected by the vendor for high $\mathrm{dR} / \mathrm{dT}$ at $50 \mathrm{mK}$.

To recycle the ADR, the first step is to magnetize the two stages by commanding their control setpoints to a value above the helium tank temperature. As stage 2 warms above the tank, heat switch HS2 is powered on, and as stage 1 warms above stage 2, heat switch HS1 is powered on. Closed-loop control eventually results in both magnets reaching their peak current of $2 \mathrm{amps}$, and both stages cooling as they equilibrate with the tank. When stage 2 cools below a preset threshold $(1.4 \mathrm{~K})$, heat switch HS2 is powered off, and stage 2's setpoint is gradually lowered so that it steadily cools stage 1. When stage 1 cools below its preset threshold of $0.75 \mathrm{~K}$, heat switch HS1 is powered off.

Stages 1 and 2 are then demagnetized by commanding their control setpoints to the desired hold temperatures, $50 \mathrm{mK}$ and $0.5 \mathrm{~K}$ respectively. After stable control is obtained, the stages are steadily demagnetized to maintain temperature as they absorb heat. The ADRC can be set so that the recycling process repeats only when commanded from the ground, or automatically when the current in stage 1 falls below a preset threshold $(5 \mathrm{~mA})$.

\subsection{ADR Recycling}

Figures $3 \mathrm{a}$ and $3 \mathrm{~b}$ show the currents and temperatures of stages 1 and 2 during a typical recycle. In contrast to the previous statement that the recycling procedure does not depend on starting condition, the time does. The amount of heat that must be rejected from the salt pills depends on the starting currents and temperatures. Except for the initial cycle after launch where the salt pills start at the bath temperature and zero current, the stages will start at the hold 
temperatures $(50 \mathrm{mK}$ and $0.5 \mathrm{~K})$ and non-zero current. The salt pills will have absorbed an amount of heat proportional to the duration of the previous hold, and the time needed to reject heat will also be proportional. All other operations, such as magnetizing from low to high temperature and vice versa, are essentially fixed duration. In practice, a recycle can be completed in as little as 30 minutes, but for the vast majority of cases where the previous hold runs close to completion, it takes just over 45 minutes.
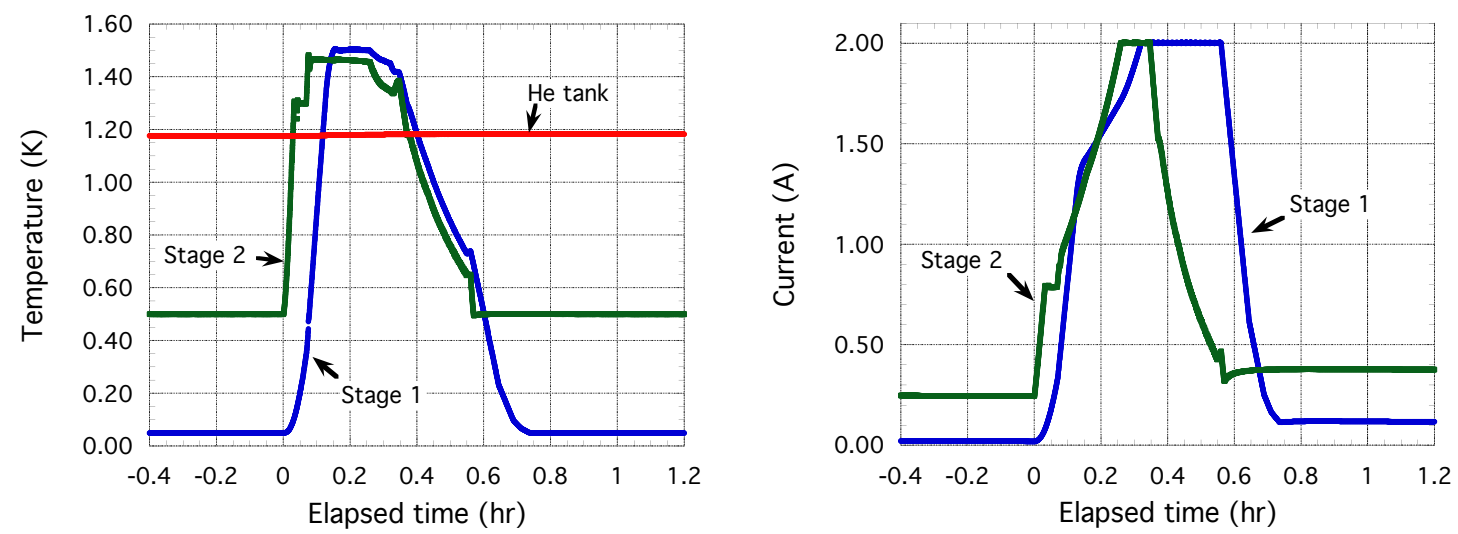

Figure 3. Temperatures (left) and magnet currents (right) during an ADR recycle.

After a recycle, the instrument requires an equilibration period during which temperatures and heat flows stabilize within the ADR, the detector array and its housing, and the detectors reach their ultimate resolution. Of particular concern is ohmic heating at the inner vapor cooled shield (IVCS), where the ADR's magnet leads transition from resistive wire to high-temperature superconductor. This warms the IVCS and can lead to a long-term drift in the heat flow from the detector array to the stage 1 salt pill. The dependency arises from thermal coupling of the detectors' JFET amplifiers to the IVCS, and changes in thermal gradients within the detector array as the conducted heat load through lead wires stabilizes. The resistive portion of the magnet leads was optimized based on the duty cycle each stage, which for stage 1 is very low, with the result that during a typical recycle the IVCS warms from $\sim 22 \mathrm{~K}$ to $\sim 25 \mathrm{~K}$, and recovers with a $\sim 2$ hour time constant.

The effect of the IVCS temperature fluctuation could not be definitively characterized during ground tests, as the helium temperature was typically warmer (1.2-1.3 K) and the porous plug bypass valve was open, leading to much greater vapor cooling and faster recovery of the IVCS after a recycle. In spite of this, it was found that detector equilibration on orbit was only slightly longer than that observed during ground tests, and the time required was 20-25 minutes. The equilibration time is determined by the rms noise on the thermometry, and the difference between ground and space is believed due to additional thermometry noise from high-energy particle hits. This additional measurement noise had no appreciable effect on detector resolution.

\subsection{ADR Hold Time and SXS Observing Efficiency}

After demagnetizing to their hold temperatures, both stages are steadily demagnetized as they absorb heat. For stage 1, the ADRC controls temperature using a germanium resistance thermometer mounted on the detector array, and a proportional-integral feedback loop that regulates magnet voltage. After a short equilibration period, the heat loads on both salt pills are essentially constant, varying slowly as the helium tank recovers from the heat input during recycling.

The demagnetization curves for all 18 ADR cycles completed before Hitomi lost operation are shown in Figure 4. Nearly all of the heat load on stage 1 is conducted through components whose warm end is at the helium tank temperature - less than $2 \%$ is conduction through heat switch HS1. As an aggregate, the heat load is proportional to $T_{\text {tank }}^{2.138}$, and therefore steadily decreased as the helium cooled after launch. This is seen as a progressive elongation of the hold time from the first cycle, when the helium was $1.4 \mathrm{~K}$, until the last, when the helium had stabilized at $1.125 \mathrm{~K}$. For the latter cycles, the hold time is seen to be just under 48 hours. 
For single-shot coolers, there is typically a requirement for duty cycle - the fraction of time that a cooler is at its (low) operating temperature. A more relevant metric is the instrument's observing efficiency - the fraction of time that the detectors are both cold and stable enough for science measurements - which, for SXS, is a shared requirement among the detector, ADR and electronics subsystems. In practice, the time from the start of a recycle to full detector stability was typically 1 hour, and the interval between the start of commanded recycles was 48 hours. This yields an observing efficiency of nearly $98 \%$, well in excess of the $90 \%$ requirement.

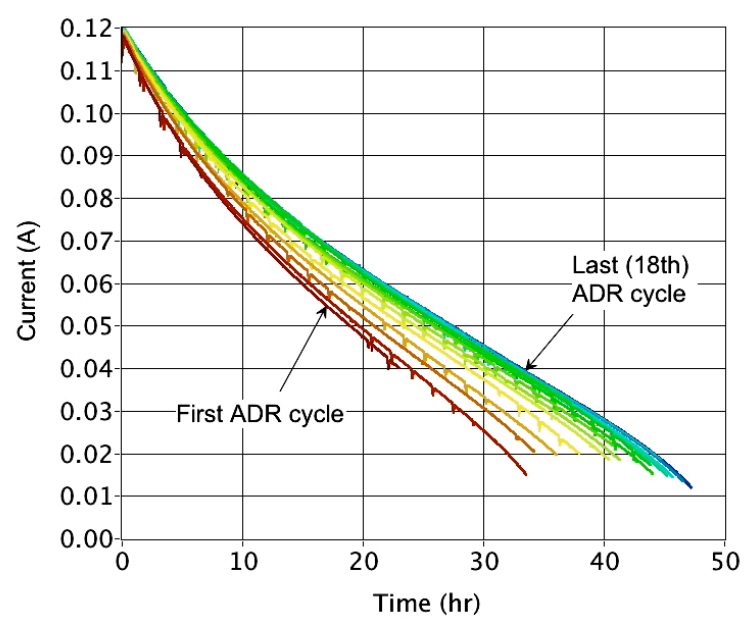

Figure 4. Demagnetization curves for stage 1 for the 18 cycles completed on orbit. Time is from the first crossing of 50 $\mathrm{mK}$ after recycling. The exponential approach of the helium tank to its steady-state temperature gave rise to a gradual increase in hold time with each cycle.

One notable feature of the demagnetization curves is periodic dips in current that correlated with the observatory passing through the South Atlantic Anomaly (SAA). Each day, roughly half of the satellite's $\sim 15$ orbits pass through some portion of the SAA, subjecting the SXS to an excess of high-energy particles for up to 15 minutes. The effect is seen more clearly in the detector array temperature (Figure 5), which exhibits infrequent, random pulses outside the SAA, but an almost continuous stream of pulses within the SAA. The primary effect appears to be high-energy particle strikes within the thermometers' sensing element, as the temperature rise is far larger than would be expected from energy deposited as particles traversed the salt pill or any other cold mass, and the temperature recovers within seconds. The negative temperature excursions result from the control system responding to the temperature spikes and demagnetizing the stage to keep the (apparent) temperature at $50 \mathrm{mK}$. This reaction creates the dips in magnet current in Figure 4.

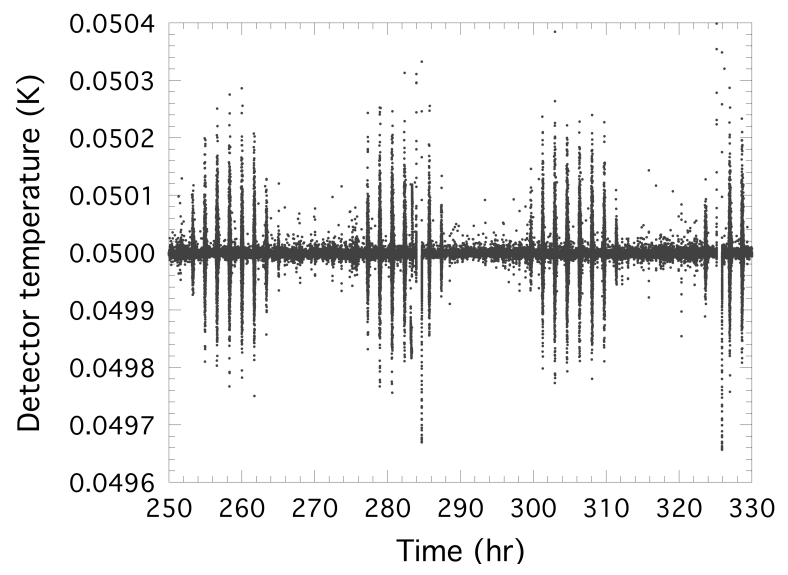

Figure 5. Detector array temperature showing the effect of high-energy particle heating during passes through the South Atlantic Anomaly. The horizontal axis is elapsed time from launch. 
Although the temperature and magnet current appear to recover after the observatory exits the SAA, there is a cumulative energy deposition that results in a slight offset in current, and an increase in the salt pill's time-average heat load. On long (many orbit) time scales, the increase is $30-40 \mathrm{nW}$. Figure 6 shows the heat load on stage 1 as determined from fitting the demagnetization curves in Figure 4 to curves calculated assuming constant heat loading. The solid line represents the heat loads measured during ground testing at the same helium tank temperature.

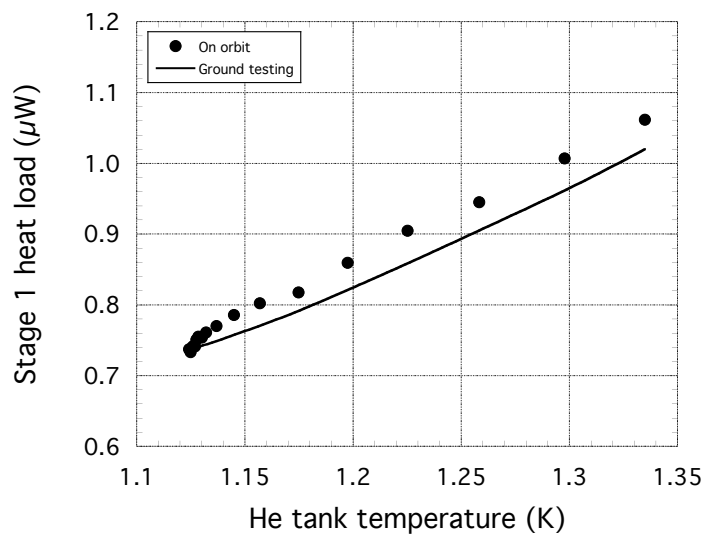

Figure 6. Heat load on stage 1 as a function of helium tank temperature for each cycle on orbit, compared to heat loads seen during ground tests.

\subsection{Liquid Mass Gauging Using Heat Rejected from the ADR}

Each time the ADR is recycled, heat is rejected to the liquid helium from three sources: heat expelled from the salt pills, hysteresis heat from the superconducting magnets and magnetic shields, and power needed to operate the heat switches. For a typical recycle that starts when the ADR is near the end of a hold period, the contributions are: $6.5 \mathrm{~J}$ from the salt pills, $3.9 \mathrm{~J}$ from magnet hysteresis, and $0.8 \mathrm{~J}$ from heating the heat switch getters, for a total of about $11.2 \mathrm{~J}$. During the subsequent hold time, only a small amount of hysteresis heat $(<0.5 \mathrm{~J})$ continues to be generated within the magnets and shields. The heat rejected during recycling warms the helium, which then recovers during the hold period. Regular cycling of the ADR gives rise to the distinctive sawtooth pattern in tank temperature shown in Figure 7.

The total heat output during an entire cycle is about $11.7 \mathrm{~J}$. Averaging over the 48-hour interval between recycles gives a time-average heat load to the liquid helium of $0.067 \mathrm{~mW}$.

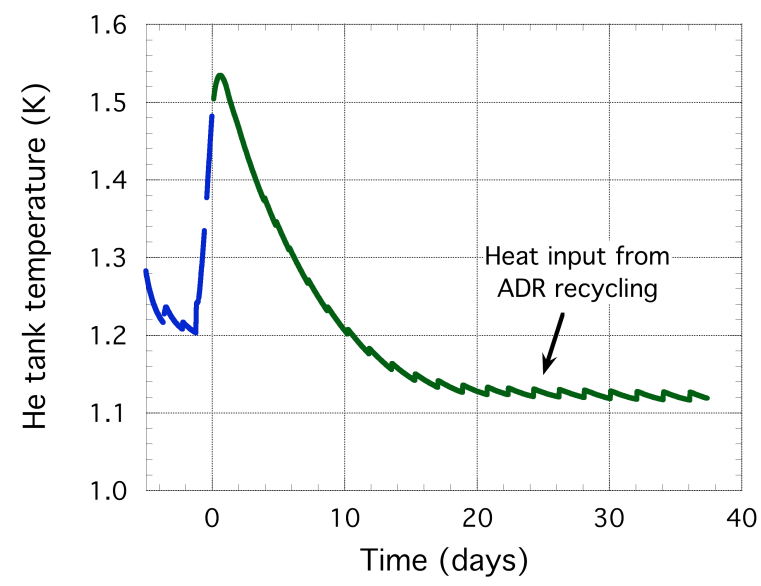

Figure 7. Helium tank temperature from shortly before launch through to the end of operations on day 38 . The increase leading up to launch was due to closure of the vent valve and periodically turning off the cryocoolers. 
During the ground test campaign for the SXS instrument, a thermodynamic model of the $\mathrm{ADR}^{14}$ was constructed so that the heat generation rate as a function of time could be calculated. The original purpose was to determine the timeaverage heat load from the ADR in order to verify it met the requirement of $<0.20 \mathrm{~mW}$ (see Table 1), but it also provided a mechanism for mass gauging the helium tank during the mission. The basic mass gauging process ${ }^{15}$ involves measuring the temperature change in a volume of liquid produced by a known heat input, and using the thermodynamic properties of superfluid helium to calculate the amount of liquid present. The main challenge arises from the fact that the heat input and the temperature change are not instantaneous. This can be resolved by measuring temperature drift rates before and after the heat input, and extrapolating to obtain the temperature difference at the midpoint of the heat input.

Direct mass gauging usually involves a short duration ( $\sim 100$ seconds) heat pulse, requiring temperature extrapolation over relatively short time periods, during which the before and after cooling rates are essentially constant. The strategy employed for Hitomi was to use the integrated heat output during ADR recycles, along with fore and aft temperature drifts extrapolated to the time at which half the heat was introduced. The measurement has two major uncertainties associated with it.

The first is the accuracy of the ADR thermal model. An uncertainty of $0.1 \mathrm{~J}$ in total heat generation translates to a mass uncertainty of about 0.6 liter. Consequently, the ADR's thermal model was based on direct calorimetric measurements of heat flow during various operations, such as constant voltage ramps of each magnet, and these were calibrated by comparing the temperature increase during these operations to the liquid's response to a known heat input (using a heater).

The second arises from the extended duration of the ADR mass gauging process, $\sim 1$ hour. In particular, the aft temperature drift rate must be extrapolated backward in time by tens of minutes, during which the cooling rate of the tank cannot be assumed constant. However, since the time constant for tank cooling is about 4 days, the cooling rate should vary by less than $0.5 \%$ over the measurement time, and the linear approximation is reasonable.

The mass gauge analysis for the ADR cycles conducted during the 38 days of Hitomi's operating life is shown in Figure 8. The mass values are shown compared to estimates of the liquid volume based on direct measurement using a superconducting level gauge immediately after the pre-launch topoff, and integrating the boiloff losses during subsequent ground operations and mass loss inferred from cooling of the liquid on orbit.

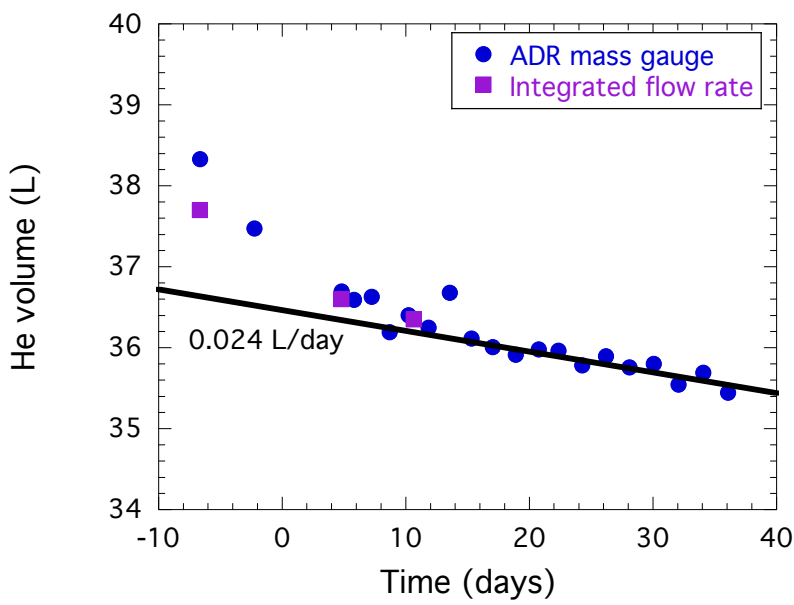

Figure 8. Helium volume as determined from ADR recycles, compared to a direct measurement after the final top-off that was then integrated to reflect boiloff loss as the helium cooled. The solid line represents a loss rate of $0.024 \mathrm{~L} / \mathrm{day}$.

The best fit to the liquid volume after equilibrium temperature was reached, at about day 10 , indicated a steady mass loss of $0.024 \pm 0.0022$ liters/day. Under these conditions, which would persist as long as the cryocoolers remained operational, the liquid carried to orbit had a predicted lifetime of 4.2 years. 


\section{SUMMARY}

The SXS instrument on the Astro-H x-ray observatory was launched cold, carrying approximately 36 liters of superfluid helium to orbit on February 17, 2016. On ascent, the valve that allows venting of the helium gas through the porous plug was opened ${ }^{16}$. During the first two days on orbit, the Stirling and Joule-Thomson cryocoolers were powered on, thus reducing heat loads on the liquid helium and accelerating its cooling to the point where ADR operations could begin.

On day 5, as the helium cooled below $1.4 \mathrm{~K}$ and the first ADR cycle was initiated by ground command. The first recycle took somewhat longer than usual - about 1 hour - as both salt pill's started warmer $(1.4 \mathrm{~K})$ than for subsequent recycles, requiring the rejection of more heat. The cycle was successful and the detectors stabilized quickly to $50 \mathrm{mK}$. X-ray events were then recorded both from external sources and an on-board Fe-55 calibration source. The detector resolution for all pixels was eventually verified to be the same as before launch, $\sim 4.9 \mathrm{eV}^{17,18,19}$.

All subsequent ADR recycles were commanded from the ground either as the ADR approached the end of its hold period, or earlier if needed for observatory commissioning activities. These activities included early observations of high-intensity celestial targets to verify proper detector operation before opening the dewar's gate valve to give the detector array an unobstructed view of the x-ray telescope. With the gate valve closed, the detector array could observe $\mathrm{x}$-rays from the telescope only through a thin beryllium window that stops $\mathrm{x}$-rays less than about $2 \mathrm{keV}$.

In all, 18 ADR cycles were conducted during the 38 days on orbit that the observatory was operational. Cooling of the helium to a final temperature of $1.125 \mathrm{~K}$ steadily increased the ADR's hold time to approximately 48 hours. Recycling required approximately 45 minutes, yielding a duty cycle of $98.5 \%$. When an equilibration time for the detectors to stabilize at $50 \mathrm{mK}$ after a recycle is included ( $\sim 25$ minutes), the SXS instrument achieved an observing efficiency for the entire 38 day period of just under $98 \%$.

Unfortunately, starting on day 38 , a series of attitude control issues resulted in the observatory entering into an uncontrolled spin, from which recovery is judged impossible. The last telemetry received shows Hitomi spinning with the solar arrays pointed away from the sun, but all systems within the SXS still operating normally.

\section{REFERENCES}

[1] Mitsuda, K., et al., "The high-resolution x-ray microcalorimeter spectrometer system for the SXS on ASTRO-H", Proc. SPIE 7732, 773211-773211-10 (2010).

[2] Takahashi, T., et al., "The ASTRO-H Mission", Proc. SPIE 7732, 77320Z-77320Z-18 (2010).

[3] Mitsuda, K., et al. "The high-resolution x-ray microcalorimeter spectrometer, SXS, on Astro-H”, J. Low Temp. Phys. 167, 795-802 (2012).

[4] Soong, Y., Okajima, T., Serlemitsos, P. J., Odell, S. L., Ramsey, B. D., et al., “ASTRO-H Soft X-ray telescope (SXT)," Proc. SPIE 9144 (2014).

[5] Fujimoto, R., et al. "Cooling system for the soft X-ray Spectrometer (SXS) onboard ASTRO-H”, Proc. SPIE , 7732, $77323 \mathrm{H}-1-7$ (2010).

[6] Yoshida, S., et al. "Flight model performance test results of a helium dewar for the soft X-ray spectrometer onboard ASTRO-H", Cryogenics, 74, 10-16 (2016).

[7] Sato, Y., et al. "Development status of the mechanical cryocoolers for the soft x-ray spectrometer on board AstroH," Cryogenics 64, 182-188 (2014).

[8] Shirron, P. J., Kimball, M. O., Wegel, D. C., and Miller, F., "ADR design for the soft x-ray spectrometer instrument on the Astro-H mission", Cryogenics 50, 494-499 (2010).

[9] Shirron, P. J., et al., "Design and predicted performance of the 3-Stage ADR for the soft x-ray spectrometer instrument on Astro-H." Cryogenics 52, 165-171 (2012).

[10] Shirron, P. J., Kimball, M. O., James, B., Muench, T., DiPirro, M. J., Bialas, T., et al., "Operating modes and cooling capabilities of the 3-stage ADR developed for the soft x-ray spectrometer instrument on Astro-H," Cryogenics 74, 2-9 (2016). 
[11] Sneiderman, G. A., et al., "Cryogen-free operation of the soft x-ray spectrometer instrument," in Proc. SPIE, 990599 (2016).

[12] Tsujimoto, M., et al., "In-orbit operation of the ASTRO-H SXS," in Proc. SPIE, 9905-31 (2016).

[13] Source of supply for Cernox-1030 and GR-200A-30: LakeShore Cryotronics Inc., 575 McCorkle Blvd., Westerville, OH 43082 USA.

[14] Shirron, P. J., et al., "Thermodynamic performance of the 3-stage ADR for the astro-h soft x-ray spectrometer instrument," Cryogenics 74, 24-30 (2016).

[15] See, for example, Volz, S. M., DiPirro, M. J., and Ryschkewitsch, M. G., "A calorimetric mass gauge system for the cosmic background explorer (COBE)," Adv. in Cryo. Eng. 35, 1703-1709 (1990).

[16] Ezoe, Y., et al., "Porous plug phase separator and superfluid film suppression system for the soft x-ray spectrometer onboard ASTRO-H," in Proc. SPIE, 9905-28 (2016).

[17] Kelley, R. L., et al., "The ASTRO-H high-resolution soft x-ray spectrometer," in Proc. SPIE, 9905-28 (2016).

[18] Porter, F. S., et al., "In-flight performance of the soft x-ray spectrometer detector system on ASTRO-H," in Proc. SPIE, 9905-29 (2016).

[19] Kilbourne, C. A., et al., "The design, implementation, and performance of the ASTRO-H SXS calorimeter array and anti-coincidence detector," in Proc. SPIE, 9905-97 (2016). 